\title{
Prophylactic DC Vaccination Controls Pancreatic Cancer Growth in a Mouse Model
}

Anna Shangguan ${ }^{1,2}$, Na Shang ${ }^{1}$, Matteo Figini ${ }^{1}$, Liang Pan ${ }^{1,3}$, Jia Yang ${ }^{1}$, Quanhong Ma1 ${ }^{1}$ Su Hu ${ }^{1,4}$, Aydin Eresen ${ }^{1}$, Chong Sun ${ }^{1,5}$, Bin Wang ${ }^{1,6}$, Yuri Velichko ${ }^{1,7}$, Vahid Yaghmai ${ }^{1,7}$, Zhuoli Zhang ${ }^{1,7}$

1 Department of Radiology, Feinberg School of Medicine, Northwestern University, Chicago, IL, USA

2 Medical Student Training Program, Northwestern University, Chicago, IL, USA

3 Department of Radiology, The Third Affiliated Hospital of Suzhou University, Changzhou, Jiangsu, China

4 Department of Radiology, The First Affiliated Hospital of Soochow University, Suzhou, Jiangsu, China

5 Department of Orthopaedics, The Affiliated Hospital of Qingdao University, Qingdao, Shandong, China

6 Department of General Surgery, Nanfang Hospital, Southern Medical University, Guangdong Provincial Engineering Technology Research Center of Minimally Invasive Surgery, Guangzhou, China

7 Robert H. Lurie Comprehensive Cancer Center, Northwestern University, Chicago, IL, USA

Running Title: Prophylactic IP DC-vaccination prevents PDAC tumor growth.

Keywords: DC vaccines, pancreatic cancer, prevention, MRI

\section{Corresponding Author Contact Information:}

Zhuoli Zhang

Northwestern University

Department of Radiology

737 N Michigan Ave, $16^{\text {th }}$ Floor 
Chicago, IL 60611 USA

Phone: (312)695-5753

Fax: (312)926)-5991

E-mail: zhuoli-zhang@northwestern.edu

Financial Support: This study was supported by the National Cancer Institute (grants R01CA209886, R01CA196967), by 2019 Harold E. Eisenberg Foundation Scholar Award and by the Fischel Fellowship Award at the Robert H. Lurie Comprehensive Cancer Center.

Conflicts of interest disclosure: The authors declare no potential conflicts of interest. 


\section{Translational Relevance}

Pancreatic ductal adenocarcinoma (PDAC) is the $4^{\text {th }}$ leading cause of cancer-related deaths with high recurrence after surgery due to a paucity of effective post-surgical adjuvant treatments. DC-vaccines can activate multiple anti-tumor immune responses but have not been explored for PDAC treatment. No standard delivery route has also been established for DC vaccination, but intraperitoneal (IP) delivery is of particular interest because it allows increased DC-vaccine dosage and thus migration to draining lymph nodes. Here, we for the first time showed that IP delivery of DC-vaccines prior to tumor induction decreased tumor volume and prolonged survival in a PDAC mouse model. These approaches may be readily translated clinically for PDAC treatment as both DC vaccination and IP delivery are already used at the bedside for treatment or in clinical trials. 


\section{Abstract}

Purpose: Pancreatic ductal adenocarcinoma (PDAC) is the $4^{\text {th }}$ leading cause of cancer-related deaths with high recurrence after surgery due to a paucity of effective post-surgical adjuvant treatments. DC-vaccines can activate multiple anti-tumor immune responses but have not been explored for post-surgery PDAC recurrence. Intraperitoneal (IP) delivery may allow increased DCvaccine dosage and migration to lymph nodes. Here, we investigated the role of prophylactic DC vaccination controlling PDAC tumor growth with IP delivery as an administration route for DC vaccination.

Methods: DC-vaccines were generated by ex vivo differentiation and maturation of bone marrow derived precursors. 20 mice were divided into 4 groups $(n=5)$ and treated with DC-vaccines, unpulsed mDCs, Panc02 lysates, or no treatment. After tumor induction, mice underwent $3 \mathrm{MRI}$ scans to track tumor growth. Apparent diffusion coefficient (ADC), a quantitative MRI measurement of tumor microstructure, was calculated. Survival was tracked. Tumor tissue was collected after death and stained with HE, Masson's' Trichrome, TUNEL, and anti-CD8 stains for histology.

Results: DC-vaccinated mice demonstrated stronger anti-tumor cytotoxicity compared with control groups on LDH assay. DC-vaccine mice also demonstrated decreased tumor volume, prolonged survival, and increased $\triangle A D C$ compared with control groups. On histology, DC-vaccine group had increased apoptosis, increased CD8+ $T$ cells, and decreased collagen. $\triangle A D C$ negatively correlated with \% collagen in tumor tissues.

Discussion: Prophylactic DC vaccination may inhibit PDAC tumor growth during recurrence and prolong survival. $\triangle A D C$ may be a potential imaging biomarker that correlates with tumor histologic features.

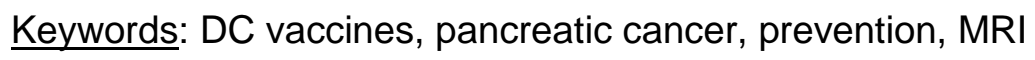




\section{Introduction}

Pancreatic ductal adenocarcinoma (PDAC) is the $4^{\text {th }}$ leading cause of cancer mortality in the US, and 5-year survival in patients with PDAC remains as low as $8 \%$ in US (1). Surgical resection is currently the only curative treatment, but less than $20 \%$ of patients with PDAC are eligible for initial resection (2). PDAC recurrence rate after surgical resection is up to $60 \%$ in the first year, and 5-year survival of patients who underwent complete resection is only $25 \%$ (3). Nonsurgical therapies for PDAC include systemic chemotherapies (4-7), molecularly-targeted therapies (8), conventional ablative therapies (9-13), and radiotherapy (14-17); however, they have so far offered little or no survival benefit $(4,6,7,11,17)$.

In recent years, dendritic cell (DC) vaccination has emerged as a novel and potential adjuvant treatment for PDAC. DCs are antigen-presenting cells (APCs) with the unique capacity to activate naive antigen-specific T cells into cytotoxic T cells, prime NK cells, and induce other anti-tumor immune responses (18-20). Autologous DCs matured and loaded with tumor antigens (DC-vaccines) ex vivo have been investigated for immunotherapy of many cancer types including pancreatic cancer (21-23). Clinical studies strongly established that DC-vaccines are safe in patients and can induce long-lasting robust antitumor immune responses, but long-term benefits of DC-vaccines were reported in only scattered patients (24-26). DC-vaccines have not fulfilled their promise.

The long-term and robust anti-tumor immune responses elicited by DC vaccines suggest that DC vaccination may also inhibit cancer growth and prevent recurrence after initial treatment. Bauer et al demonstrated that DC-vaccines in combination with gemcitabine prevent cancer recurrence in a Panc02 xenograft PDAC mouse model $(27,28)$. DC-vaccines have also been demonstrated to prevent the recurrence of ovarian cancer, sarcomas, and hepatocellular carcinoma (29-32). The tumor-preventative effects of DC vaccines were mediated through DC activation of CD8+ T cells (30) in a dose-dependent manner (33), similar to the mechanism of DC-vaccines in cancer treatment. In early-phase clinical trials, however, only scattered patients 
demonstrated diminished cancer recurrence, prolonged survival, or other responses to therapy (34). DC vaccination for prevention of cancer development and recurrence still require further investigation.

As priming and activation of T cells by DCs primarily take place in draining lymph nodes (LNs), sufficient DC-vaccine migration from the vaccination site to the $L N$ is a critical requirement for DC vaccination in cancer treatment and prevention. The administration route of DC-vaccines is directly related with the strength of subsequent antitumor immune response and immune memory $(35,36)$. Subcutaneous (SC) injection, intra-tumor (IT) injection, and intra-LN (IN) injection have been used in preclinical research and clinical trials $(37,38)$. However, only a small number of DCs can migrate to LNs and organs by subcutaneous injection $(37,38)$, and direct delivery of DCs to LN/tumor by IN/IT injection diminishes DC therapeutic function $(37,38)$. Given that peritoneal LNs and the spleen comprise the most important secondary lymphoid organs (39) in the abdomen, we have recently tested DC delivery by intraperitoneal injection (IP). Our studies demonstrated that IP injection improves DCs migration to abdominal LNs and can be directly translated to clinical settings (40).

MRI may allow early prediction of responses to therapy such as the formation of a robust immune response, diminished tumor growth, and prolonged survival. Immune responses to DC vaccination are mediated by lymphocyte infiltration of tumor and tissue edema on histology. As a well-established quantitative real-time non-invasive imaging tool, diffusion weighted-MRI (DWMRI) is of particular interest in pancreatic cancer imaging as it is sensitive to the microscopic mobility of water molecules, known as Brownian motion, which in turn is related to the microstructural features of the imaged tissues (41). The apparent diffusion coefficient (ADC) is the most common quantitative metric derived from DW-MRI. ADC values may detect treatmentrelated changes soon after therapy to help predict early response to treatment (42-44). DW-MRI is particularly appealing from a practical standpoint as it provides microstructural information related to pathology without the need for intravenous or oral contrast agents. 
The purposes of this study were a) to investigate prophylactic IP delivery of DC-vaccines for clinical translation; b) to investigate the role of preventive $\mathrm{DC}$ vaccination in the treatment and prevention of PDAC; and c) to investigate the relationship between changes in ADC and treatment outcomes following DC vaccination. We hypothesized that $\mathrm{DC}$ vaccination prior to tumor induction will lead to diminished tumor growth due to enhanced antitumor immunity, tumor inflammation, and increased immune cell infiltration into PDAC tumors.

\section{Materials/Methods}

Cell lines and media. The Panc02 mouse cell line is derived from amethylcholanthreneinduced PDAC tumors in C57BL/6 mice and was purchased from the American Type Culture Collection (ATCC; Rockville, MD). Panc02 was cultured in 2 mM L-glutamine RPMI media supplemented with $10 \%$ fetal bovine serum, $100 \mu \mathrm{g} / \mathrm{mL}$ penicillin, and $100 \mu \mathrm{g} / \mathrm{mL}$ streptomycin (Gibco, Waltham, MA). All cells were maintained in a humidified environment with $5 \% \mathrm{CO}_{2}$ at $37^{\circ} \mathrm{C}$. Cell viability was checked by trypan blue staining before UV irradiation and tumor induction in mouse models.

General animal procedures. All procedures concerning animals are approved by the institutional animal care and use committee (IACUC) of Northwestern University. C57BL/6 mice were obtained from Jackson Lab (Bar Harbor, ME). Anesthesia was induced and maintained in C57BL/6 mice by inhalation of isoflurane $(2 \%, 1 \mathrm{~L} / \mathrm{min}$, Patterson Veterinary, Greeley, CO) for imaging and surgical procedures.

UV irradiation of Panc02 cells. Panc02 cells were trypsinized, washed, and diluted with PBS to a concentration of $6 \times 10^{6}$ cells $/ \mathrm{mL} .500 \mu \mathrm{L}$ of diluted Panc02 cells was placed in a 6-well plate to cover the bottom of each well. At least $0.75 \mathrm{~J} / \mathrm{cm}^{2}$ of UV-B light was applied to each well (minimum 10 min UV radiation exposure with lamp placed $1 \mathrm{~cm}$ from cells). Plate was rocked 
halfway through irradiation to ensure even UV irradiation. Viability was checked with trypan blue staining. Irradiated panc02 lysates were added to 8-day old immature DCs for antigen pulsing.

Flow cytometry and monoclonal antibodies. Bone marrow derived DCs (BMDCs) were obtained from mice using the protocols described below. After 8 days of in vitro culture, BMDCs

were washed with cold PBS, blocked with FcR blocker, and then stained with PerCP-CY ${ }^{\mathrm{TM}} 5.5$ CD11c monoclonal antibody (mAb), APC-CD86 mAb (all from BD Bioscience, San Jose, CA), PE MHC-II mAb (Southern, Biotech, Birmingham, AL), and appropriate isotype controls at $2 \mu \mathrm{g} / 3 \mathrm{x}$ $10^{5}$ cells by incubation for 40 min at $4^{\circ} \mathrm{C}$. After washing with PBS with $10 \% \mathrm{FBS}$, surface markers were analyzed via fluorescence-activated cell sorting (FACS, BD LSRFortessa ${ }^{\mathrm{TM}}$ cell analyzer, San Jose, CA). Flow data was analyzed using FlowJo (Ashland, OR).

Generation and antigen-loading of bone marrow derived DCs. BMDCs were generated using previously described protocols (28). Briefly, dendritic cell precursors were collected from bone marrow of the femur and tibia of C57BL/6 mice. Precursor cells $\left(6 \times 10^{5}\right.$ cells $/ \mathrm{mL}$ ) were incubated in RPMI media with L-glutamine, FBS, penicillin, streptomycin, interleukin 4 (IL-4, $1 \mathrm{ng} / \mathrm{mL}$, Shenandoah Biotechnology, Warwick, PA), and granulocyte macrophage colony stimulating factor (GM-CSF, 10ng/mL, Shenandoah Biotechnology, Warwick, PA) for differentiation into immature DCs (iDCs). iDCs, found as loosely adherent cells, were matured and loaded with tumor antigens by co-incubation with Panc02 cell lysates (10:1 DCs:Panc02 cells), interferon-y (IFN-y, $100 \mathrm{ng} / \mathrm{mL}$, Shenandoah Biotechnology, Warwick, PA), and bacterial lipopolysaccharides (LPS, $250 \mathrm{ng} / \mathrm{mL}$, Sigma-Aldrich, St. Louis, MO) for $24 \mathrm{~h}$. Panc02 lysates were generated by UV irradiation at $750 \mathrm{~mJ} / \mathrm{cm}^{2}$ as described above. Non-vaccine $\mathrm{mDC}$ s were generated by culturing iDCs in the presence of LPS and IFN- $\gamma$ without Panc02 lysates. FACS analysis was used to verify DC maturity.

Tumor induction in a Panc02 orthograft mouse model. Panc02 cells were used to induce orthotopic pancreatic tumors in C57BL/6 mice using previously published protocols (28). Briefly, Panc02 cells were trypsinized, washed with PBS, and concentrated to $3 \times 10^{5}$ cells/3.75 
$\mu \mathrm{L}$ in PBS. Matrigel was added to Panc02 cell solution at matrigel:Panc02 cell solution ratio of 1:3. The resulting solution was vortexed thoroughly to generate a homogeneous mixture. $A 1 \mathrm{~cm}$ longitudinal incision was made in the left upper quadrant to access the pancreas. $3 \times 10^{5}(5 \mu \mathrm{L})$ cells were slowly injected into the tail of the pancreas. The needle was kept in the injection site for $10 \mathrm{sec}$ and then slowly removed to prevent Panc02 cell leakage from the injection site. The incision was closed with a 2-layer suture. Antibiotic gel and analgesics were applied daily for 3 days according to IACUC protocols. Post-operative condition was monitored for 1 week.

Vaccination Strategies. 6 week old mice $(n=20)$ of similar age were randomly divided into 4 groups ( $n=5$ for each group): DC vaccines, unpulsed mDCs, Panc02 lysates, and no treatment. Mice in the 3 treatment groups received weekly IP injections for 3 weeks of $9 \times 10^{6} \mathrm{DC}$ vaccines, $9 \times 10^{6}$ unpulsed $\mathrm{mDCs}$, or $9 \times 10^{5}$ Panc02 cell lysates, while the no treatment group received no injections. 1 week after the final IP injection, all mice underwent tumor induction using protocols described above. Tumor growth and survival were monitored by weekly MRI scans for 3 weeks. All mice were euthanized after reaching physiologic endpoint to collect tumor tissue samples for histology. Vaccination strategy is illustrated in Figure 1A.

In vivo cytotoxicity assay. LDH release assay was performed according to manufacturer instructions (Pierce ${ }^{\mathrm{TM}}$ LDH Cytotoxicity Assay Kit, Pierce Biotechnology, Waltham, MA) to assess anti-tumor cytotoxicity of CD8+ cells after DC vaccination or other treatments. Briefly, 3 groups ( $n=3$ for each group) were given only one dose of $9 \times 10^{6} \mathrm{DC}$ vaccines, $9 \times 10^{6}$ unpulsed mDCs, or no treatment via IP injection. All mice underwent tumor induction 1 week after the last treatment using previously described methods. 1 week after tumor induction, all mice were euthanized to collect the spleen for immune cell isolation. CD8+ cells were isolated from immune cells in the spleen by negative selection using a commercially available mouse CD8+ cell isolation kit (Miltenyi Biotec, Auburn, CA) according to manufacturer's instructions. CD8+ cells and Panc02 cells were incubated together in a 96 well plate, with 3000 Panc02 cells per well and CD8+:Panc02 cell ratios of 5:1, 10:1, 20:1, and 40:1. Control groups including 
Panc02 cell only (high control), CD8+ cell only, and media only (low control), were also plated. The 96-well plates were incubated for 4 hours. Wells containing only Panc02 cells (high controls) were incubated with a lysis solution for $15 \mathrm{~min}$. The LDH substrate was added to each well, and the plate was incubated for an additional $30 \mathrm{~min}$. The substrate stop solution was then added, and well absorbance was then immediately measured using a plate reader. \% cytotoxicity was calculated using relative absorbances of test and control wells:

$\%$ cytotoxicity $=\frac{A_{\text {sample }}-A_{\text {high control }}-A_{C D \text { o only }}-A_{\text {low control }}}{A_{\text {high control }}-A_{\text {low control }}}$, where $A$ is absorbance.

MRI Image Acquisition. All images were acquired using a 7.0 T small-animal MRI scanner with a commercial rat brain surface coil (ClinScan, Bruker Biospin). All animals underwent image acquisition starting 1 week after tumor induction to confirm successful tumor induction in mouse models and to track tumor growth. Tumor growth was monitored for 3 weeks after tumor induction. Image acquisition parameters are described in Table 1.

Image Analysis. All anatomic images were analyzed using ITK-SNAP (version 3.6, University of Pennsylvania), an open source software. Tumor volumes were calculated by tracing free-hand regions of interest (ROIs) around the tumor margins on each slice of axial or coronal T2W images containing the implanted tumor. Diffusion-weighted images were first postprocessed using Matlab (Mathworks, Natick, MA) to generate ADC maps. A freehand ROI was drawn around the margins of the tumor in one slice of ADC map using axial T2W images as reference. Regions of necrosis and artifacts were avoided. Change in tumor ADC ( $\triangle A D C)$ were calculated by the following equation: $\triangle A D C=A D C_{3 w}-A D C_{1 w}$, where $A D C_{1 w}$ and $A D C_{3 w}$ are the tumor ADC measured at 1 week and 3 weeks after tumor induction, respectively. The tumor growth rate $r$ was calculated using the exponential tumor growth model, $r=\frac{\ln \left(V_{T 2}\right)-\ln \left(V_{T 1}\right)}{T_{2}-T_{1}}$, where $V_{T 2}$ and $V_{T 1}$ are the tumor volumes at the second and first time point respectively, $T_{2}$ and $T_{1}$ are the times between tumor induction and the $2^{\text {nd }}$ or first imaging time point in days. 
Histology. All animals were euthanized at physiologic endpoint according to IACUC guidelines. Pancreatic tumor tissue was dissected and fixed in formalin immediately after euthanasia. Tumor tissues were stained with hematoxylin-eosin (HE), Masson's Trichrome, and terminal deoxynucleotidyl transferase dUTP nick end labeling (TUNEL) stains as well as labeled with CD8+ antibodies to detect the presence of cytotoxic T lymphocytes in the targeted tumor tissues. All images were examined using ImageJ. Quantitative measurements were made by blinded researchers. Collagen fibers were quantified in 3 representative fields at $200 x$ magnification in all slides stained with Masson's Trichrome. Tumor tissue apoptosis was identified on TUNEL-stained slices and quantified as the percentage of TUNEL-positive cells/total cells in 3 representative fields at $200 x$ magnification randomly selected from each slice. CD8+ cells were identified and quantified in 3 representative fields at 200x magnification randomly selected from each slice. Apoptosis, fibrosis, and CD8+ cells will be compared amongst all groups.

Statistical Analysis. All statistical analyses were performed using the software GraphPad Prism (GraphPad Software, La Jolla, CA). One-way analysis of variance (ANOVA) was performed on all sample tumor sizes for all control groups at each time to determine whether tumor sizes differed across groups for each time point. ANOVA was also used to compare tumor growth rates amongst all groups and between time points. Kaplan-Meier analysis was performed on survival of animals across all groups. Linear regression was performed to determine correlations between tumor volume and survival, growth rates and tumor volume, and tumor growth rates and survival. For $\triangle A D C$ values, one-way ANOVA was performed to compare $\triangle A D C$ values for each group. Linear regression was performed on survival, tumor volume, and tumor growth rate vs $\triangle A D C$. For all studies, $\mathrm{P}<0.05$ or lower was considered significant.

\section{Results}

\section{Generation of PDAC-specific DC vaccines.}


Cells differentiated from BMDCs were assessed for DC purity and maturity using FACS. Mature DCs were identified as MHCI+/CD11C+/CD86+, while immature DCs were identified as MHCII+/CD11C+/CD86-. After culturing in RPMI with IL-4 and GM-CSF, DCs were found to stain negatively for CD86 and positively for MHC-II and CD11c; $86 \%, 98 \%$, and $91 \%$ of DCs stained positively for CD86, CD11C, and MHC-II, respectively, when cultured with Panc02 lysates as well as IFN- $\mathrm{y}$, LPS, IL-4, and GM-CSF (Figure 1B). Mature DCs also demonstrated an increased MHC-II median fluorescence intensity (MFI, 8819) compared with immature DCs (3655). Mature DC vaccines can thus be differentiated from bone marrow derived precursors at a high purity.

\section{In vivo CTL response after prophylactic DC-vaccination via IP injection}

Figure 1C shows cell-mediated LDH release at CD8+ (effector) cells to Panc02 (target) cells ratios of $5: 1,10: 1,20: 1$, and 40:1. CD8+ cells were extracted from mice that underwent only

one dose of DC vaccines. Panc02 cells incubated with CD8+ cells from DC vaccine group demonstrated increased cytotoxicity compared with the unpulsed mDCs and no treatment groups at both $20: 1$ (39\% vs $11 \%$ and $9 \%$, respectively, $\mathrm{P}<0.05)$ and $40: 1(42 \%$ vs $13 \%$ and $10 \%$, respectively, $P<0.05) E: T$ ratios. Evidently, mice receiving even a single dose of $D C$ vaccines developed a strong anti-tumor immune response compared with control group.

\section{Animal tumor growth and survival after prophylactic DC vaccination}

Representative T2 axial images of the induced tumor are shown in Figure 2A. All tumors were visible on MRI by 1 week after tumor challenge regardless of treatment group and increased in size over time. On T2W images, tumors were clearly demarcated from surrounding tissue and were generally hyperintense compared with normal pancreatic tissue. Ascites could be observed in mice at as early as 3 weeks after tumor induction.

On average, mice receiving DC vaccines demonstrated significantly decreased $(P<0.05)$ tumor volumes at all 3 time points $\left(5.39,15.2\right.$, and $23.4 \mathrm{~mm}^{3}$ for 7,14 , and $21 \mathrm{~d}$, respectively) compared with mice receiving unpulsed $\mathrm{mDCs}\left(15.0,20.5\right.$, and $\left.56.2 \mathrm{~mm}^{3}\right)$, Panc02 lysates (14.6, 31.1, and $\left.52.7 \mathrm{~mm}^{3}\right)$, or no treatment $\left(29.1,45.5,129 \mathrm{~mm}^{3}\right)$ (Figure 2B-C). DC vaccinated mice 
also demonstrated significantly prolonged survival $(P<0.01)$ compared with control groups, with median survivals of $61 \mathrm{~d}$ for DC vaccines group, $49 \mathrm{~d}$ for the unpulsed mDCs group, $40 \mathrm{~d}$ for the Panc02 lysates group, and $35 \mathrm{~d}$ for the no treatment group (Figure 2D). Linear regression comparing animal survival with tumor volumes at 3 weeks after tumor challenge demonstrated a significant negative correlation $\left(R^{2}=0.4001, P<0.005\right.$, Figure $\left.2 E\right)$. Prophylactic IP delivery of DC vaccines was able to inhibit tumor growth and prolong survival in a Panc02 mouse model.

At 7-14 d, DC vaccines demonstrated the greatest growth rate at $0.1590 \mathrm{~mm}^{3} / \mathrm{d}$, followed by $0.1061 \mathrm{~mm}^{3} / \mathrm{d}$ for Panc02 lysates group, $0.07404 \mathrm{~mm}^{3} / \mathrm{d}$ for no treatment group, and 0.04421 $\mathrm{mm}^{3} / \mathrm{d}$ for $\mathrm{mDC}$ group (Figure 3A). In contrast, the no treatment group demonstrated the greatest growth rate $\left(0.1473 \mathrm{~mm}^{3} / \mathrm{d}\right)$ at $14-21 \mathrm{~d}$, followed by the $\mathrm{mDC}$ group $\left(0.1422 \mathrm{~mm}^{3} / \mathrm{d}\right)$, the Panc02 lysates group $\left(0.07576 \mathrm{~mm}^{3} / \mathrm{d}\right)$, and the DC vaccines group $\left(0.06085 \mathrm{~mm}^{3} / \mathrm{d}\right)$ (Figure $\left.3 A-B\right)$. There was significant difference between growth rates across treatment groups at both time points $(P<0.05)$. Multiple t testing demonstrated a significant decrease in growth rate for the $D C$ vaccine group (-60\%) and a significant increase in growth rate for the mDCs group (+249\%) (Figure 3A). No significant changes in growth rates were observed in the Panc02 lysates and no treatment groups (Figure 3A). No significant correlation $\left(R^{2}=0.1259\right)$ was observed between growth rate from 14-21d and survival in general, but a significant correlation $\left(R^{2}=0.9559\right)$ was found specifically for the DC vaccinated group (Figure 3C-D).

\section{Correlation between $A D C$ values and response to $D C$ vaccination}

On ADC maps (Figure 4A), tumors were easily distinguished from surrounding structures by a decrease in signal compared with normal pancreatic tissue and an increase in signal compared with the spleen. As seen in Figure 4B, mice that received no treatment demonstrated the largest decrease in tumor $\operatorname{ADC}\left(-1.18 \times 10^{-4}, \mathrm{P}<0.05\right)$ over the course of observation, followed by the Panc02 lysates group $\left(-9.65 \times 10^{-5}\right)$, the $\mathrm{mDC}$ group $\left(-7.95 \times 10^{-5}\right)$, and the DC-vaccine group $\left(-5.11 \times 10^{-5}\right)$. In both $\triangle \mathrm{ADC}$ vs survival and $\triangle \mathrm{ADC}$ vs $21 \mathrm{~d}$ tumor volumes plots, the 4 treatment groups (DC vaccines, unpulsed mDCs, Panc02 lysates, and no treatment) showed 
distinct clustering (Figure 4C, E). A linear correlation was found between $\triangle A D C$ and survival $\left(R^{2}\right.$ $=0.5667)$ and between $\triangle A D C$ and tumor volume $\left(R^{2}=0.4563\right)$, as seen in Figure $4 D$ and $F$.

\section{Histologic Evaluation.}

On HE stained sections (Figure 5A), all tumor tissues were dense and desmoplastic and demonstrated loss of exocrine secretory structures. Masson's Trichrome stain of tumor tissues showed a high collagen density in the tumor, confirming the presence of dense fibrotic stroma in the tumor tissue that is characteristic of PDAC. In addition, tumor tissues showed the presence of cells staining positively for TUNEL and CD8, demonstrating cell death and immune cell infiltration, respectively, in the tumor tissue. Together, these basic images indicated that Panc02 orthograft tumors closely mimic human PDAC pathology and that C57BL/6 mice can form an antitumor immune response against the implanted tumor.

Tissue collagen and cells staining positively for TUNEL and CD8 were quantified on all slides (Figure 5B-G). Tumors from mice treated with DC vaccines demonstrated greater numbers of CD8+ cells per field in the tumor tissue $(131, P<0.05)$ compared with tumors from mice treated with unpulsed mDCs (36 per field), Panc02 lysates (37 per field), and no treatment (34 per field). This further confirmed that mice receiving prophylactic DC vaccines exerted a stronger anti-tumor immune response compared with control groups. Mice treated with DC vaccines also demonstrated the greatest percentage of cells staining positively for TUNEL $(9.87 \%, P<0.05)$ compared with the mDC group (1.59\%), the Panc02 lysates group (2.30\%), or no treatment group (1.29\%). DC vaccinated mice thus demonstrated greater tumor cell apoptosis and tumor tissue death compared with control groups. On Masson's Trichrome, animals prophylactically treated with DC vaccines had the least percentage area of collagen fibers $(16.4 \%, P<0.05)$ compared to animals treated with unpulsed mDCs (41.1\%), Panc02 lysates (43.0\%), or no treatment (51.3\%), suggesting that DC-vaccinated animals demonstrated decreased tumor fibrosis compared with un-vaccinated animals. Linear regression comparing pooled percentage collagen on Masson's Trichrome stain and $\triangle A D C$ demonstrated a significant negative correlation $\left(R^{2}=0.4685, P<0.05\right.$, 
Figure $5 \mathrm{H})$. Together, $\mathrm{DC}$ vaccine was further confirmed to generate a stronger immune response with increased tumor tissue death and decreased tumor fibrosis.

\section{Discussion}

While many studies have previously explored DC vaccination for treatment of pancreatic cancers $(27,45-49)$, few studies have investigated the role of DC vaccines for prevention of tumor development and/or recurrence. In this study, we used a murine model of PDAC to show that clinically translatable preventative intraperitoneal DC vaccination can effectively diminish tumor growth and that ADC can be used as an imaging biomarker for assessment of preventive DC vaccination therapeutic responses. Pathology results also showed that tumor collagen density on histology correlated with the number of CD8+ cells and apoptosis.

Although DC vaccines have been extensively studied for pre-surgical or non-surgical management of many types of cancer, few studies have investigated their potential for prevention of cancer relapse or recurrence, especially in pancreatic cancer. As the post-surgical PDAC recurrence rate is high even administration of adjuvant therapies, development of effective postsurgical adjuvant therapeutic approaches is critical for PDAC treatment. Bauer et al. demonstrated in that treatment with tumor-lysate-pulsed DC-vaccines plus gemcitabine led to a complete response and also prevented tumor recurrence after successful initial treatment in a Panc02 mouse model via SC delivery, which only allows delivery of a small number of DCs (28). Moreover, DC vaccines have not been studied extensively as a potential stand-alone therapy for prevention of tumor recurrence via IP delivery. In this study, we showed that prophylactic DC vaccination can inhibit tumor growth and prolong survival, suggesting that stand-alone DC vaccination may be an effective adjuvant treatment in the clinical setting.

While we observed strong correlation between tumor volume at 21 days, $\triangle A D C$, and survival, no correlation was observed between growth rates and other quantitative measurements. Furthermore, a strong positive relationship between growth rate and survival was observed in 
only the treatment group, suggesting that early rapid tumor growth is correlated with improved response to treatment. This contradicts classical responses to treatment where slowed growth rates lead to improved survival, as was seen in responses to chemotherapy and radiotherapy. Additional processes such as pseudoprogression may be involved in initial pancreatic tumor growth. Pseudoprogression, where inflammation, edema, and transient immune cell infiltration into tumor tissue may cause initial increase in tumor volume, is often observed in patients soon after treatment with immunotherapeutic agents such as checkpoint inhibitor agents. In our study, mice in the DC vaccination group demonstrated the strongest immune response against tumor cells. Moreover, the DC-vaccine group, which elicits the strongest anti-tumor immune response as seen on CTL assay, showed that rapid tumor growth at early time points correlated with improved response to therapy. In addition, the DC vaccination group demonstrated the highest initial growth rate and then the lowest growth rates at sequential time points. DC vaccination group also demonstrated the lowest tumor volume at 3 weeks and longest survival. In addition, we found that the DC vaccination group had significantly increased CD8+ cell infiltration in the tumor stroma. All together, these details further support that tumor pseudoprogression may contribute to the rapid initial tumor growth in the DC vaccine group and better response to therapy.

We also observed that $\triangle \mathrm{ADC}$ values differed across treatment groups, with $\mathrm{DC}$ vaccine group having the highest $\triangle \mathrm{ADC}$ and no treatment group having the lowest $\triangle \mathrm{ADC} . \triangle \mathrm{ADC}$ correlated significantly with percent collagen on Masson's Trichrome stain as well as with tumor volume at $21 \mathrm{~d}$ and animal survival. Our results are consistent with previous findings of correlations between ADC measurements and tumor tissue architecture. For example, Zhang et al. demonstrated in a rat model that ADC measurements correlated with pancreatic cancer microstructure (50). Wang et al. further demonstrated that denser fibrotic stroma in pancreatic cancer correlated with lower $\triangle \mathrm{ADC}(51)$. In this study, we demonstrated that $\mathrm{DC}$ vaccination correlated with increased $\triangle \mathrm{ADC}$, which in turn correlated with decreased fibrosis on histology, decreased tumor volume, and prolonged survival. This suggests that DC vaccination may inhibit 
the formation of dense fibrotic tumor stroma, leading to increased susceptibility to anti-tumor immune cells. As many other treatment methods depend on infiltration into the tumor stroma, DC vaccination may potentiate the efficacy of other tumor treatments that require tumor penetration such as chemotherapy and checkpoint inhibition therapy when used in combination. We thus further confirmed $\triangle A D C$ as an imaging marker with correlations to histology that has the potential to predict survival and response to therapy in the clinical setting.

This study has several limitations. First, the number of animals per study group was small. However, the number of animals chosen per treatment group was the minimal number of animals necessary to achieve sufficient power based on statistical analysis. Additional animals may be used for each treatment group for future studies. Second, tumor growth was tracked at only 3 time points $-7,14$, and 21 days after tumor induction. These three time points are among the most frequent time points used in tracking tumor growth via in vivo MR imaging studies. For future studies, additional time points may be used to track tumor growth over longer periods of time. Third, we did not track whether mouse models demonstrated an elevation of cytokines such as IFN-y and IL-2 to confirm the presence of a robust immune response. However, we have shown that CD8+ T cells of DC-vaccinated mice can specifically induce cell death of Panc02 cancer cells using an LDH cell-mediated cytotoxicity assay, a well-established method for assessing in vivo anti-tumor immune response. For future studies, additional markers of anti-tumor response may be measured as a secondary confirmation of an in vivo anti-tumor immune response. Lastly, we did not directly compare outcomes after IP delivery with that of other modes of DC vaccine delivery within our study. However, other modes of administration, such as SC and IT delivery, have been extensively studied as delivery methods for DC vaccines, and we have shown that tumor volume and survival results from IP delivery in our study is superior to that by other modes of delivery (28).

In summary, we demonstrated prophylactic DC vaccination as a powerful tool that may inhibit the development of pancreatic tumors, and we have also shown that $\triangle A D C$ may be a 
potentially powerful prognostic imaging biomarkers that has correlations with tumor histologic features. Furthermore, clinically applicable approaches developed in this study can be directly translated to clinical practice. 


\section{References}

1. Cancer Stat Facts: Pancreatic Cancer NIH NCl Surveilance, Epidemiology, and End Results $\begin{array}{lllll}\text { Program2018 } & \text { [September } & \text { 17. 2018] } & \text { Available from: }\end{array}$ https://seer.cancer.gov/statfacts/html/pancreas.html.

2. Bilimoria KY, Bentrem DJ, Ko CY, Ritchey J, Stewart AK, Winchester DP, Talamonti MS. Validation of the 6th edition AJCC Pancreatic Cancer Staging System: report from the National Cancer Database. Cancer. 2007;110(4):738-44. doi: 10.1002/cncr.22852. PubMed PMID: 17580363.

3. Wilkowski R, Wolf M, Heinemann V. Primary advanced unresectable pancreatic cancer. Recent Results Cancer Res. 2008;177:79-93. PubMed PMID: 18084950.

4. Von Hoff DD, Ervin T, Arena FP, Chiorean EG, Infante J, Moore M, Seay T, Tjulandin SA, Ma WW, Saleh MN, Harris M, Reni M, Dowden S, Laheru D, Bahary N, Ramanathan RK, Tabernero J, Hidalgo M, Goldstein D, Van Cutsem E, Wei X, Iglesias J, Renschler MF. Increased survival in pancreatic cancer with nab-paclitaxel plus gemcitabine. N Engl J Med. 2013;369(18):1691-703. doi: 10.1056/NEJMoa1304369. PubMed PMID: 24131140; PMCID: PMC4631139.

5. Ychou M, Viret F, Kramar A, Desseigne F, Mitry E, Guimbaud R, Delpero JR, Rivoire M, Quenet F, Portier G, Nordlinger B. Tritherapy with fluorouracil/leucovorin, irinotecan and oxaliplatin (FOLFIRINOX): a phase II study in colorectal cancer patients with non-resectable liver metastases. Cancer Chemother Pharmacol. 2008;62(2):195-201. doi: 10.1007/s00280-0070588-3. PubMed PMID: 17901955.

6. Assenat E, Desseigne F, Thezenas S, Viret F, Mineur L, Kramar A, Samalin E, Portales F, Bibeau F, Crapez-Lopez E, Bleuse JP, Ychou M. Cetuximab plus FOLFIRINOX (ERBIRINOX) as first-line treatment for unresectable metastatic colorectal cancer: a phase II trial. Oncologist. 2011;16(11):1557-64. doi: 10.1634/theoncologist.2011-0141. PubMed PMID: 22016477; PMCID: PMC3233290. 
7. Conroy T, Desseigne F, Ychou M, Bouche O, Guimbaud R, Becouarn Y, Adenis A, Raoul JL, Gourgou-Bourgade S, de la Fouchardiere C, Bennouna J, Bachet JB, Khemissa-Akouz F, PereVerge D, Delbaldo C, Assenat E, Chauffert B, Michel P, Montoto-Grillot C, Ducreux M, Groupe Tumeurs Digestives of U, Intergroup P. FOLFIRINOX versus gemcitabine for metastatic pancreatic cancer. N Engl J Med. 2011;364(19):1817-25. doi: 10.1056/NEJMoa1011923. PubMed PMID: 21561347.

8. Amanam I, Chung V. Targeted Therapies for Pancreatic Cancer. Cancers (Basel). 2018;10(2). doi: 10.3390/cancers10020036. PubMed PMID: 29382159; PMCID: PMC5836068.

9. Ruarus A, Vroomen L, Puijk R, Scheffer H, Meijerink M. Locally Advanced Pancreatic Cancer: A Review of Local Ablative Therapies. Cancers (Basel). 2018;10(1). doi: 10.3390/cancers10010016. PubMed PMID: 29320420; PMCID: PMC5789366.

10. Arcidiacono PG, Carrara S, Reni M, Petrone MC, Cappio S, Balzano G, Boemo C, Cereda S, Nicoletti R, Enderle MD, Neugebauer A, von Renteln D, Eickhoff A, Testoni PA. Feasibility and safety of EUS-guided cryothermal ablation in patients with locally advanced pancreatic cancer. Gastrointest Endosc. 2012;76(6):1142-51. doi: 10.1016/j.gie.2012.08.006. PubMed PMID: 23021160.

11. Girelli R, Frigerio I, Giardino A, Regi P, Gobbo S, Malleo G, Salvia R, Bassi C. Results of 100 pancreatic radiofrequency ablations in the context of a multimodal strategy for stage III ductal adenocarcinoma. Langenbecks Arch Surg. 2013;398(1):63-9. doi: 10.1007/s00423-012-1011-z. PubMed PMID: 23053459.

12. Silviu UB, Daniel P, Claudiu M, Sandulescu L, Simona F, Stefan P, Valeriu S, Adrian S. Endoscopic ultrasound-guided radiofrequency ablation of the pancreas: An experimental study with pathological correlation. Endosc Ultrasound. 2015;4(4):330-5. doi: 10.4103/23039027.170426. PubMed PMID: 26643702; PMCID: PMC4672592.

13. Changela K, Patil R, Duddempudi S, Gaduputi V. Endoscopic Ultrasound-Guided Radiofrequency Ablation of the Pancreatic Tumors: A Promising Tool in Management of 
Pancreatic Tumors. Can J Gastroenterol Hepatol. 2016;2016:4189358. doi: 10.1155/2016/4189358. PubMed PMID: 27478820; PMCID: PMC4958435.

14. Vorobyov N, Rykov I, Orlova R, Cherkashin M. Chemotherapy in combination with stereotactic body radiation therapy (SBRT) for oligometastatic pancreatic cancer. BMJ Case Rep. 2018;2018. doi: 10.1136/bcr-2018-225846. PubMed PMID: 30219780.

15. El-Bared N, Portelance L, Spieler BO, Kwon D, Padgett KR, Brown KM, Mellon EA. Dosimetric Benefits and Practical Pitfalls of Daily Online Adaptive MRI-Guided Stereotactic Radiotherapy for Pancreatic Cancer. Pract Radiat Oncol. 2018. doi: 10.1016/j.prro.2018.08.010. PubMed PMID: 30149192.

16. Lauffer DC, Kuhn PA, Kueng M, Thalmann SU, Risse G, Tercier PA, Egger B, Allal AS. Pancreatic Cancer: Feasibility and Outcome After Radiochemotherapy with High Dose External Radiotherapy for Non-resected and R1 Resected Patients. Cureus. 2018;10(5):e2713. doi: 10.7759/cureus.2713. PubMed PMID: 30079279; PMCID: PMC6067808.

17. Yang SH, Shao YY, Lin CC, Kuo SH, Cheng AL, Yeh KH. A Phase I Study of S-1-based Concurrent Chemoradiotherapy Followed by Gemcitabine and S-1 in Metastatic Pancreatic Adenocarcinoma. Anticancer Res. 2018;38(8):4805-12. doi: 10.21873/anticanres.12790. PubMed PMID: 30061252.

18. Chattopadhyay G, Shevach EM. Antigen-specific induced T regulatory cells impair dendritic cell function via an IL-10/MARCH1-dependent mechanism. Journal of immunology (Baltimore, Md : 1950). 2013;191(12):5875-84. Epub 2013/11/13. doi: 10.4049/jimmunol.1301693. PubMed PMID: 24218453; PMCID: PMC3858537.

19. Pampena MB, Levy EM. Natural killer cells as helper cells in dendritic cell cancer vaccines. Front Immunol. 2015;6:13. doi: 10.3389/fimmu.2015.00013. PubMed PMID: 25674087; PMCID: PMC4309200. 
20. Gardner A, Ruffell B. Dendritic Cells and Cancer Immunity. Trends Immunol. 2016;37(12):855-65. doi: 10.1016/j.it.2016.09.006. PubMed PMID: 27793569; PMCID: PMC5135568.

21. Garg AD, Vara Perez M, Schaaf M, Agostinis P, Zitvogel L, Kroemer G, Galluzzi L. Trial watch: Dendritic cell-based anticancer immunotherapy. Oncoimmunology. 2017;6(7):e1328341. Epub 2017/08/16. doi: 10.1080/2162402x.2017.1328341. PubMed PMID: 28811970; PMCID: PMC5543823.

22. Seledtsov VI, Goncharov AG, Seledtsova GV. Clinically feasible approaches to potentiating cancer cell-based immunotherapies. Human vaccines \& immunotherapeutics. 2015;11(4):851-69. Epub 2015/05/02. doi: 10.1080/21645515.2015.1009814. PubMed PMID: 25933181; PMCID: PMC4514272.

23. Osada T, Nagaoka K, Takahara M, Yang XY, Liu CX, Guo H, Roy Choudhury K, Hobeika A, Hartman Z, Morse MA, Lyerly HK. Precision cancer immunotherapy: optimizing dendritic cellbased strategies to induce tumor antigen-specific T-cell responses against individual patient tumors. Journal of immunotherapy (Hagerstown, Md : 1997). 2015;38(4):155-64. Epub 2015/04/04. doi: 10.1097/cji.0000000000000075. PubMed PMID: 25839441.

24. Bol KF, Schreibelt G, Gerritsen WR, de Vries IJ, Figdor CG. Dendritic Cell-Based Immunotherapy: State of the Art and Beyond. Clinical cancer research : an official journal of the American Association for Cancer Research. 2016;22(8):1897-906. Epub 2016/04/17. doi: 10.1158/1078-0432.ccr-15-1399. PubMed PMID: 27084743.

25. Chang JH, Jiang Y, Pillarisetty VG. Role of immune cells in pancreatic cancer from bench to clinical application: An updated review. Medicine (Baltimore). 2016;95(49):e5541. doi: 10.1097/MD.0000000000005541. PubMed PMID: 27930550; PMCID: PMC5266022.

26. Maccalli C, Parmiani G, Ferrone S. Immunomodulating and Immunoresistance Properties of Cancer-Initiating Cells: Implications for the Clinical Success of Immunotherapy. Immunological 
investigations. 2017;46(3):221-38. Epub 2017/03/14. doi: 10.1080/08820139.2017.1280051. PubMed PMID: 28287848.

27. Bauer C, Sterzik A, Bauernfeind F, Duewell P, Conrad C, Kiefl R, Endres S, Eigler A, Schnurr M, Dauer M. Concomitant gemcitabine therapy negatively affects DC vaccine-induced CD8(+) Tcell and B-cell responses but improves clinical efficacy in a murine pancreatic carcinoma model. Cancer Immunol Immunother. 2014;63(4):321-33. doi: 10.1007/s00262-013-1510-y. PubMed PMID: 24384835.

28. Bauer C, Bauernfeind F, Sterzik A, Orban M, Schnurr M, Lehr HA, Endres S, Eigler A, Dauer M. Dendritic cell-based vaccination combined with gemcitabine increases survival in a murine pancreatic carcinoma model. Gut. 2007;56(9):1275-82. doi: 10.1136/gut.2006.108621. PubMed PMID: 17395611; PMCID: PMC1954993.

29. Chu CS, Boyer J, Schullery DS, Gimotty PA, Gamerman V, Bender J, Levine BL, Coukos G, Rubin SC, Morgan MA, Vonderheide $\mathrm{RH}$, June $\mathrm{CH}$. Phase $\mathrm{I} / \mathrm{Il}$ randomized trial of dendritic cell vaccination with or without cyclophosphamide for consolidation therapy of advanced ovarian cancer in first or second remission. Cancer Immunol Immunother. 2012;61(5):629-41. doi: 10.1007/s00262-011-1081-8. PubMed PMID: 22021066.

30. Liu Q, Zhai B, Yang W, Yu LX, Dong W, He YQ, Chen L, Tang L, Lin Y, Huang DD, Wu HP, Wu MC, Yan HX, Wang HY. Abrogation of local cancer recurrence after radiofrequency ablation by dendritic cell-based hyperthermic tumor vaccine. Mol Ther. 2009;17(12):2049-57. doi: 10.1038/mt.2009.221. PubMed PMID: 19773743; PMCID: PMC2814395.

31. Merchant MS, Bernstein D, Amoako M, Baird K, Fleisher TA, Morre M, Steinberg SM, Sabatino M, Stroncek DF, Venkatasan AM, Wood BJ, Wright M, Zhang H, Mackall CL. Adjuvant Immunotherapy to Improve Outcome in High-Risk Pediatric Sarcomas. Clin Cancer Res. 2016;22(13):3182-91. doi: 10.1158/1078-0432.CCR-15-2550. PubMed PMID: 26823601.

32. Kayashima H, Toshima T, Okano S, Taketomi A, Harada N, Yamashita Y, Tomita Y, Shirabe $\mathrm{K}$, Maehara Y. Intratumoral neoadjuvant immunotherapy using IL-12 and dendritic cells is an 
effective strategy to control recurrence of murine hepatocellular carcinoma in immunosuppressed mice. J Immunol. 2010;185(1):698-708. doi: 10.4049/jimmunol.0900187. PubMed PMID: 20498356.

33. Ge C, Li R, Song H, Geng T, Yang J, Tan Q, Song L, Wang Y, Xue Y, Li Z, Dong S, Zhang Z, Zhang N, Guo J, Hua L, Chen S, Song X. Phase I clinical trial of a novel autologous modified-DC vaccine in patients with resected NSCLC. BMC Cancer. 2017;17(1):884. doi: 10.1186/s12885017-3859-3. PubMed PMID: 29268708; PMCID: PMC5740508.

34. Paniccia A, Merkow J, Edil BH, Zhu Y. Immunotherapy for pancreatic ductal adenocarcinoma: an overview of clinical trials. Chin J Cancer Res. 2015;27(4):376-91. doi: 10.3978/j.issn.10009604.2015.05.01. PubMed PMID: 26361407; PMCID: PMC4560736.

35. Mullins DW, Sheasley SL, Ream RM, Bullock TN, Fu YX, Engelhard VH. Route of immunization with peptide-pulsed dendritic cells controls the distribution of memory and effector T cells in lymphoid tissues and determines the pattern of regional tumor control. $J$ Exp Med. 2003;198(7):1023-34. doi: 10.1084/jem.20021348. PubMed PMID: 14530375; PMCID: PMC2194213.

36. Simon T, Fonteneau JF, Gregoire M. Dendritic cell preparation for immunotherapeutic interventions. Immunotherapy. 2009;1(2):289-302. doi: 10.2217/1750743X.1.2.289. PubMed PMID: 20635946.

37. Galati D, Zanotta S. Hematologic neoplasms: Dendritic cells vaccines in motion. Clinical immunology (Orlando, Fla). 2017;183:181-90. Epub 2017/09/06. doi: 10.1016/j.clim.2017.08.016. PubMed PMID: 28870867.

38. Jiang $\mathrm{H}$, Wang $\mathrm{Q}$, Sun $\mathrm{X}$. Lymph node targeting strategies to improve vaccination efficacy. Journal of controlled release : official journal of the Controlled Release Society. 2017. Epub 2017/08/19. doi: 10.1016/j.jconrel.2017.08.009. PubMed PMID: 28818619. 
39. Malik B, Rath G, Goyal AK. Are the anatomical sites for vaccine administration selected judiciously? Int Immunopharmacol. 2014;19(1):17-26. doi: 10.1016/j.intimp.2013.12.023. PubMed PMID: 24406427.

40. Wang B, Sun C, Wang S, Shang N, Shangguan J, Figini M, Pan L, Zhou K, Ma Q, Procissi D, Velichko Y, Yaghmai V, Li G, Zhang Z. Mouse dendritic cell migration in abdominal lymph nodes by intraperitoneal administration. American journal of translational research. 2018;10(9):2859-67. PubMed PMID: 30323872; PMCID: PMC6176238.

41. Padhani AR, Liu G, Koh DM, Chenevert TL, Thoeny HC, Takahara T, Dzik-Jurasz A, Ross BD, Van Cauteren M, Collins D, Hammoud DA, Rustin GJ, Taouli B, Choyke PL. Diffusionweighted magnetic resonance imaging as a cancer biomarker: consensus and recommendations. Neoplasia. 2009;11(2):102-25. PubMed PMID: 19186405; PMCID: PMC2631136.

42. Qin L, Li X, Stroiney A, Qu J, Helgager J, Reardon DA, Young GS. Advanced MRI assessment to predict benefit of anti-programmed cell death 1 protein immunotherapy response in patients with recurrent glioblastoma. Neuroradiology. 2017;59(2):135-45. doi: 10.1007/s00234-016-17698. PubMed PMID: 28070598; PMCID: PMC6097616.

43. Nakamura Y, Bernardo M, Nagaya T, Sato K, Harada T, Choyke PL, Kobayashi H. MR imaging biomarkers for evaluating therapeutic effects shortly after near infrared photoimmunotherapy. Oncotarget. 2016;7(13):17254-64. doi: 10.18632/oncotarget.7357. PubMed PMID: 26885619; PMCID: PMC4941385.

44. Ceschin R, Kurland BF, Abberbock SR, Ellingson BM, Okada H, Jakacki RI, Pollack IF, Panigrahy A. Parametric Response Mapping of Apparent Diffusion Coefficient as an Imaging Biomarker to Distinguish Pseudoprogression from True Tumor Progression in Peptide-Based Vaccine Therapy for Pediatric Diffuse Intrinsic Pontine Glioma. AJNR Am J Neuroradiol. 2015;36(11):2170-6. doi: 10.3174/ajnr.A4428. PubMed PMID: 26338910; PMCID: PMC4644687. 45. Miyazawa M, Iwahashi M, Ojima T, Katsuda M, Nakamura M, Nakamori M, Ueda K, Naka T, Hayata K, lida T, Yamaue H. Dendritic cells adenovirally-transduced with full-length mesothelin 
cDNA elicit mesothelin-specific cytotoxicity against pancreatic cancer cell lines in vitro. Cancer Lett. 2011;305(1):32-9. doi: 10.1016/j.canlet.2011.02.013. PubMed PMID: 21397388.

46. Bauer C, Dauer M, Saraj S, Schnurr M, Bauernfeind F, Sterzik A, Junkmann J, JakI V, Kiefl R, Oduncu F, Emmerich B, Mayr D, Mussack T, Bruns C, Ruttinger D, Conrad C, Jauch KW, Endres S, Eigler A. Dendritic cell-based vaccination of patients with advanced pancreatic carcinoma: results of a pilot study. Cancer Immunol Immunother. 2011;60(8):1097-107. doi: 10.1007/s00262-011-1023-5. PubMed PMID: 21547597.

47. Kimura Y, Tsukada J, Tomoda T, Takahashi H, Imai K, Shimamura K, Sunamura M, Yonemitsu Y, Shimodaira S, Koido S, Homma S, Okamoto M. Clinical and immunologic evaluation of dendritic cell-based immunotherapy in combination with gemcitabine and/or S-1 in patients with advanced pancreatic carcinoma. Pancreas. 2012;41(2):195-205. doi: 10.1097/MPA.0b013e31822398c6. PubMed PMID: 21792083.

48. Chen J, Guo XZ, Li HY, Liu X, Ren LN, Wang D, Zhao JJ. Generation of CTL responses against pancreatic cancer in vitro using dendritic cells co-transfected with MUC4 and survivin RNA. Vaccine. 2013;31(41):4585-90. doi: 10.1016/j.vaccine.2013.07.055. PubMed PMID: 23928463.

49. Kobayashi M, Shimodaira S, Nagai K, Ogasawara M, Takahashi H, Abe H, Tanii M, Okamoto M, Tsujitani S, Yusa S, Ishidao T, Kishimoto J, Shibamoto Y, Nagaya M, Yonemitsu Y, Therapy DCVSGatJSoIC. Prognostic factors related to add-on dendritic cell vaccines on patients with inoperable pancreatic cancer receiving chemotherapy: a multicenter analysis. Cancer Immunol Immunother. 2014;63(8):797-806. doi: 10.1007/s00262-014-1554-7. PubMed PMID: 24777613. 50. Zhang Z, Zheng L, Li W, Gordon AC, Huan Y, Shangguan J, Procissi D, Bentrem DJ, Larson AC. Quantitative functional MRI in a clinical orthotopic model of pancreatic cancer in immunocompetent Lewis rats. Am J Transl Res. 2015;7(9):1475-86. PubMed PMID: 26550449; PMCID: PMC4626411. 
51. Wang Y, Chen ZE, Nikolaidis P, McCarthy RJ, Merrick L, Sternick LA, Horowitz JM, Yaghmai V, Miller FH. Diffusion-weighted magnetic resonance imaging of pancreatic adenocarcinomas: association with histopathology and tumor grade. J Magn Reson Imaging. 2011;33(1):136-42. doi: 10.1002/jmri.22414. PubMed PMID: 21182131. 
Table 1. MRI acquisition parameters.

\begin{tabular}{|c|c|c|}
\hline Purpose & Sequences & Scan Parameters \\
\hline $\begin{array}{l}\text { Axial Anatomic } \\
\text { Images }\end{array}$ & $\begin{array}{ll}\text { 3D } & \text { T2-Weighted } \\
\text { Turbo } & \text { Spin Echo } \\
\text { (TSE) } & \end{array}$ & $\begin{array}{l}\mathrm{TR} / \mathrm{TE}=3000 / 30 \mathrm{~ms} \text {, turbo-factor }=12,384 \times 264 \text { matrix, } \\
100 \times 60 \mathrm{~mm} \text { FOV, } 100 \text { slices at } 0.5 \mathrm{~mm} \text { thickness, } 10 \\
\text { min scan time }\end{array}$ \\
\hline $\begin{array}{l}\text { Axial Diffusion- } \\
\text { Weighted } \\
\text { Imaging }\end{array}$ & DWI & $\begin{array}{l}\text { TR/TE }=2000 / 40 \mathrm{~ms}, \mathrm{FOV}=28 \times 28 \mathrm{~mm} \text {, matrix size }=82 \\
\mathrm{x} 82, \mathrm{ST}=1 \mathrm{~mm} ; \text { gap }=0 \mathrm{~mm} ; \mathrm{b}-\mathrm{value}=0 \text { and } 800 \\
\mathrm{~s} / \mathrm{mm}^{2} \text {, DWI performed in } 3 \text { orthogonal directions of } \\
\text { the diffusion gradients }\end{array}$ \\
\hline
\end{tabular}


A

C

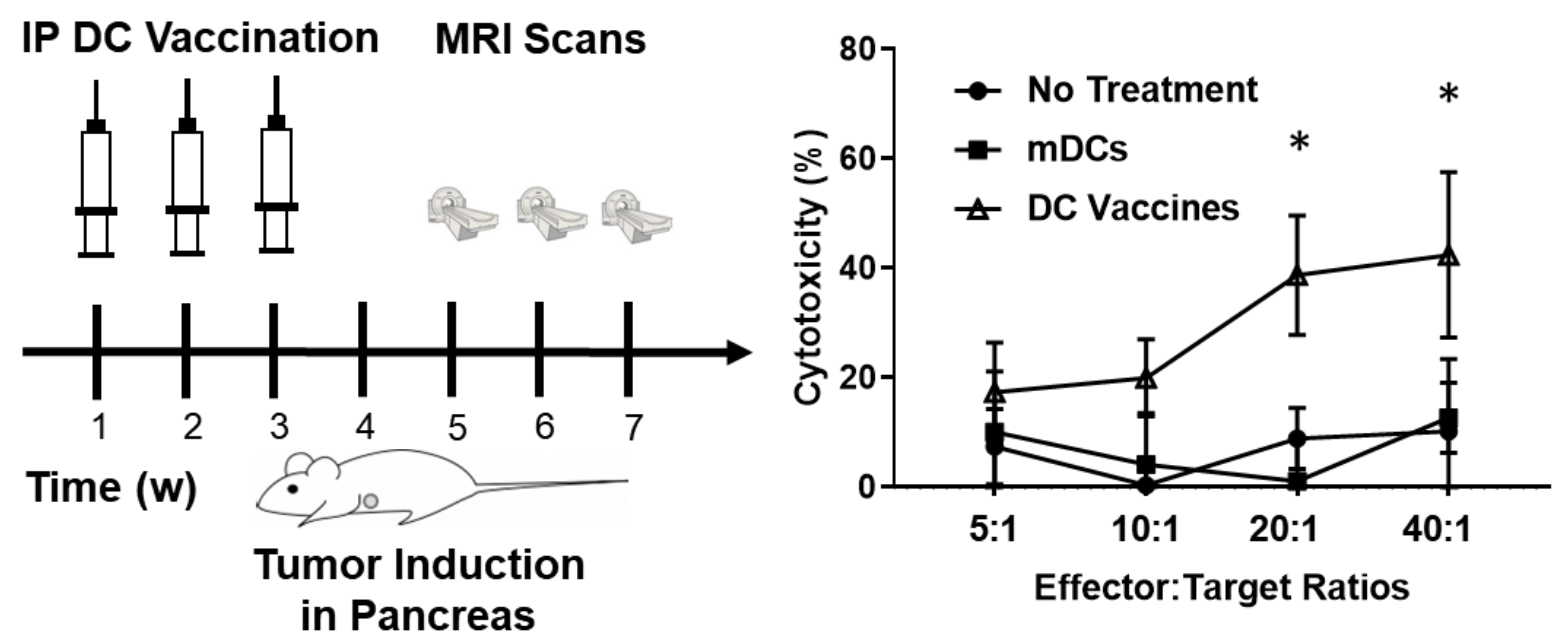

B
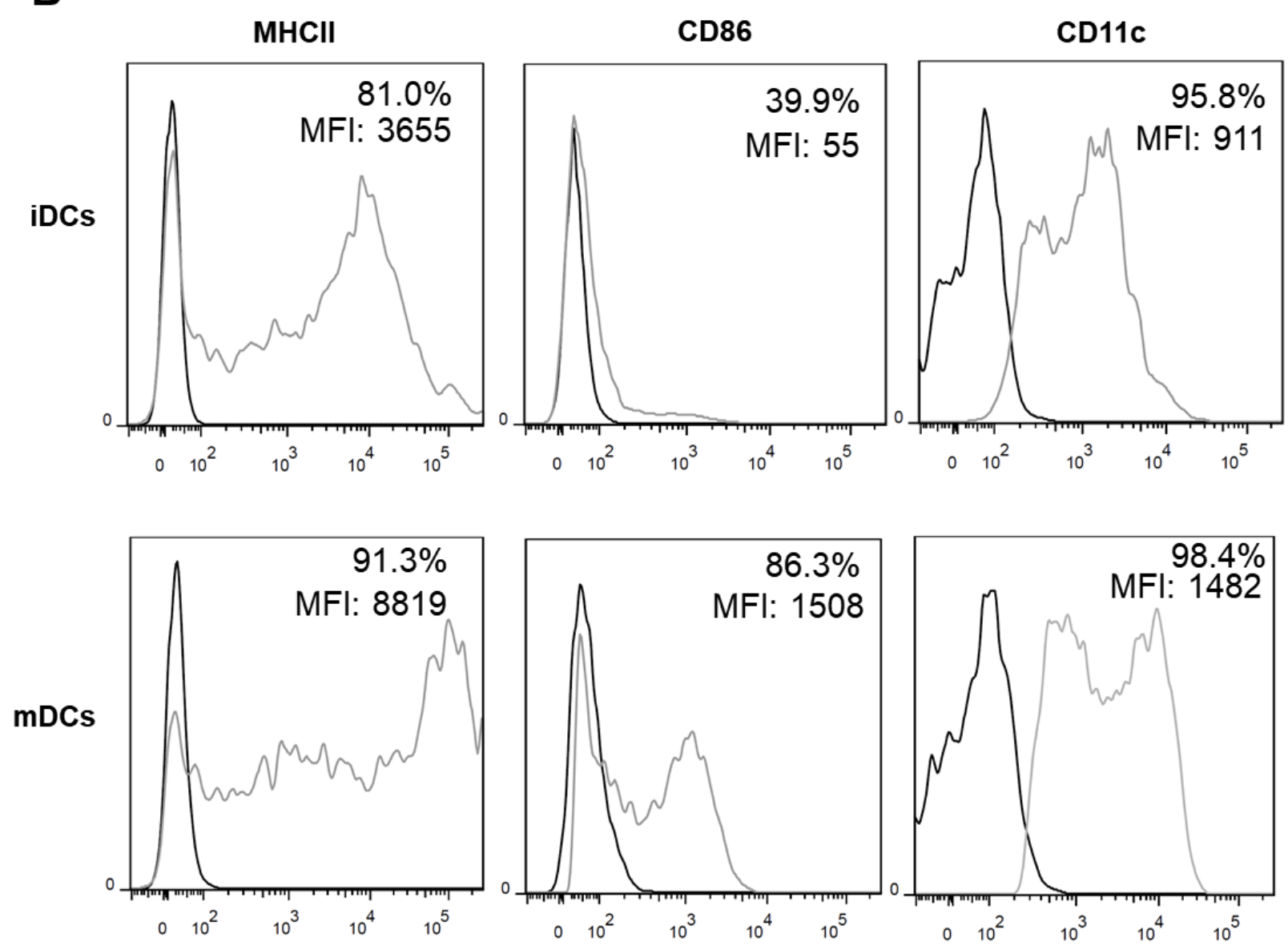

Figure 1. In vitro validation for DC vaccination. a) Representative schema of the schedule for IP injection and MRI acquisitions. All animals underwent weekly IP injections for $3 \mathrm{w}$, followed by 
tumor induction $1 \mathrm{w}$ after the last injection. Animals then underwent weekly MRI acquisition for 3 w. b) FACS analysis of immature and mature DCs. Precursor cells were extracted from mouse bone marrow and differentiated into iDCs by $6 \mathrm{~d}$ incubation in GM-CSF and IL-4. Some iDCs were collected for FACS, while others were matured in the presence of IFNy, LPS, and Panc02 lysates for FACS of mDCs. All iDCs and mDCs were stained with CD86, MHC-II, and CD11c. Only DCs that underwent maturation demonstrated high percentage that stained positively for all 3 markers, indicating maturity. Labeled percentages represent \% positive cells. MFI represents median fluorescence intensity. c) LDH assay for T cell-mediated antitumor cytotoxicity. CD8a+ cells were extracted from the spleen of mice that underwent 1 dose of DC vaccination, control treatment, or no treatment ( $\mathrm{n}=3$ for each group), followed by tumor induction. After co-incubation with Panc02 cells, LDH release by Panc02 cells were measured. Only DC-vaccinated mice demonstrated increased \% cytotoxicity at higher Effector:Target ratios $(P<.05)$. * indicates $P<0.05$. 

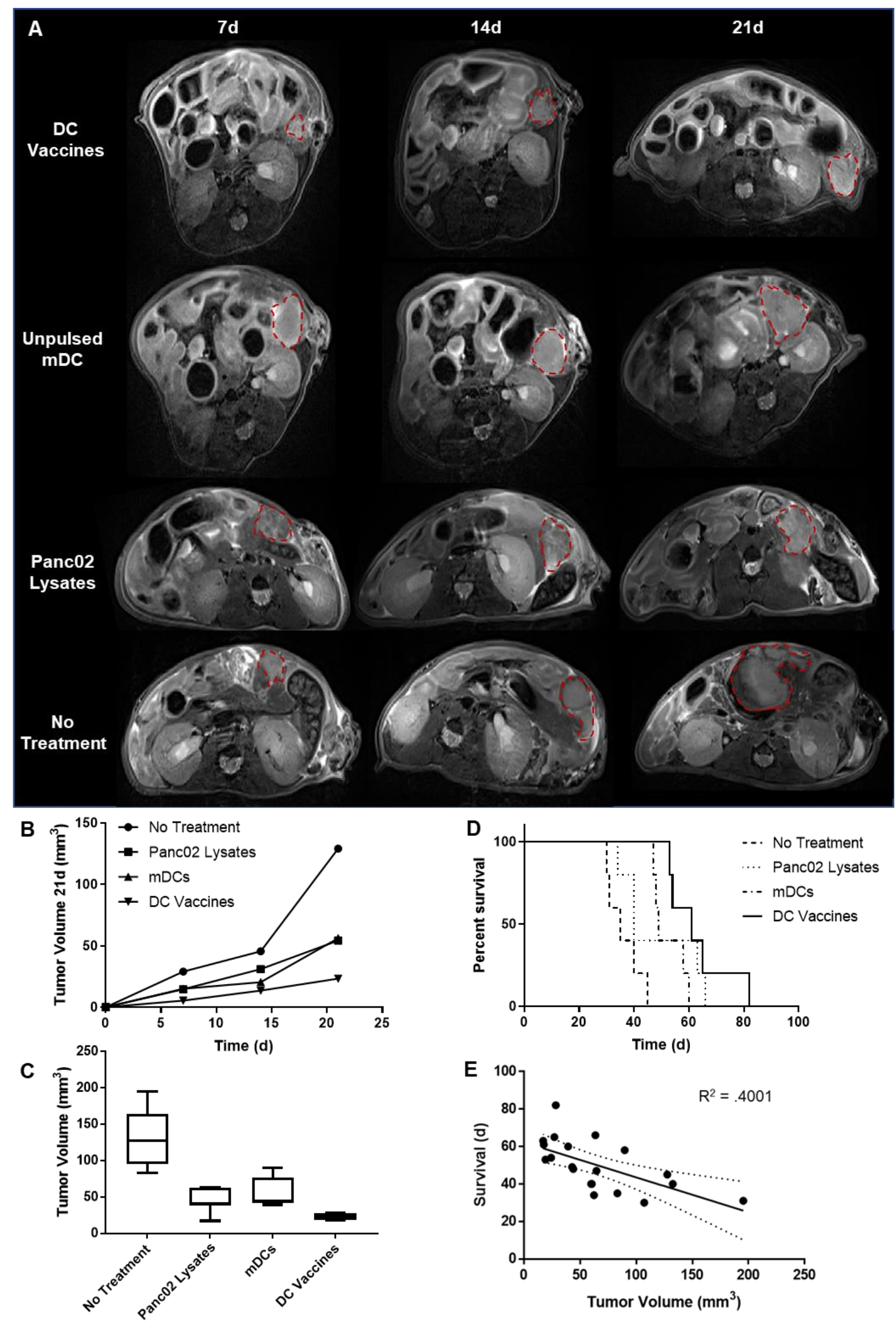
Figure 2. Representative MR images of the tumor and pancreatic tissues as well as related tumor growth and survival plots. a) Representative axial images of the pancreatic tumor (red outline) in all treatment groups. Tumors are generally hyperintense compared with normal pancreatic tissue. b) Average tumor volume $\left(\mathrm{mm}^{3}\right)$ vs. time after tumor induction (d) for all treatment and control groups ( $n=5$ for each group). c) Boxplot of tumor volume at $21 d$ for all groups ( $n=5$ for each group). Error bars contain entire range of tumor volumes for each group. Upper, middle, and lower bounds of the box represent upper quartile, median, and lower quartile, respectively, for each group. Significant difference $(P<0.05)$ was found between the tumor volumes across all 4 treatment groups at 21d using ANOVA. d) Kaplan-Meier survival curve of each treatment group ( $n=5$ or each group). Median survival of each is found in the table below the curve. DC-vaccinated group had the longest survival $(P<0.01)$, followed by unpulsed mDCs, Panc02 lysates, and no treatment groups. e) Survival (d) vs. tumor volume at $21 \mathrm{~d}\left(\mathrm{~mm}^{3}\right)$ generated from pooled animals across al treatment groups $(n=20)$. Linear regression showed a significant negative relationship $\left(R^{2}=0.4001, P<0.005\right)$. Dashed lines represent $95 \%$ confidence around the linear regression line. 
A
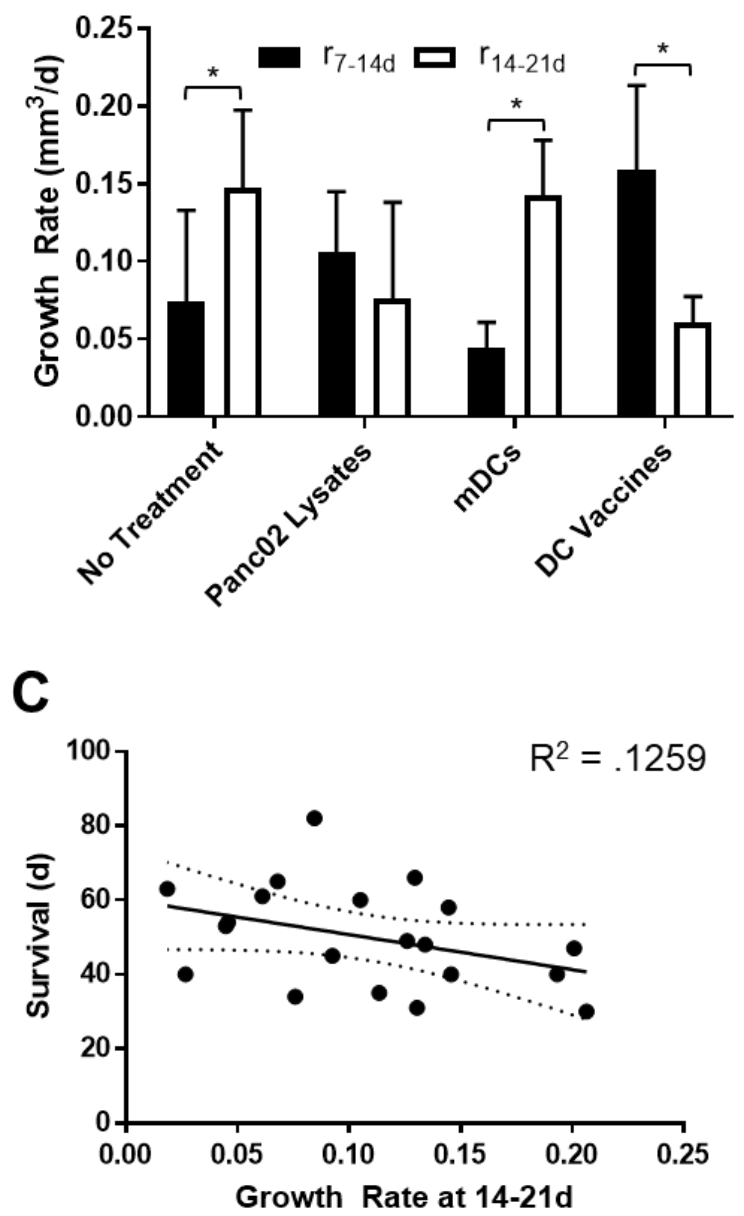

B

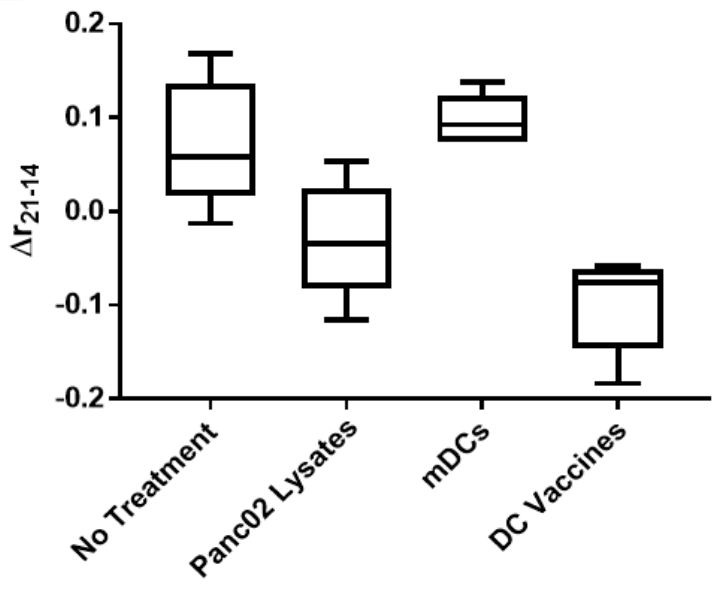

D

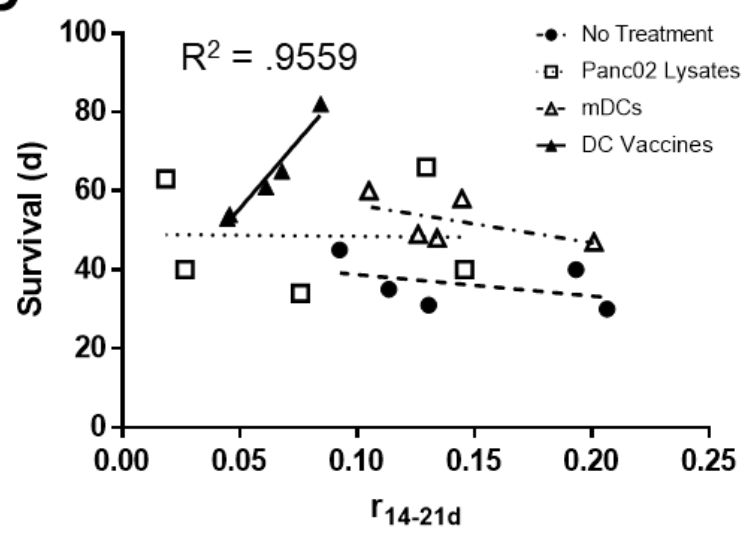

Figure 3. Comparisons of tumor growth rates calculated from tumor volumes estimated from sequential MR images. a) Tumor growth rate $\left(\mathrm{mm}^{3} / \mathrm{d}\right)$ for each treatment groups $(\mathrm{n}=5$ for each group) from 7-14 $d$ and from $14-21 d$ after tumor induction. Error bars represent \pm 1 standard deviation from the average. Significant difference $(P<0.05)$ was found among all treatment groups at each tumor growth rate time point. Tumor growth rates at each point were also significantly different for the unpulsed $m D C s$ and $D C$ vaccine groups $(P<0.05)$. b) Boxplot of changes in tumor growth rates $\left(\mathrm{mm}^{3} / \mathrm{d}\right)$ across different time points for each treatment groups $(\mathrm{n}$ = 5 for each group). Upper, middle, and lower bounds of each box represents upper quartile, median, and lower quartile, respectively, of each group. Error bars represent maximal and minimal values in each treatment group. c) Survival (d) vs growth rate from $14-21 \mathrm{~d}\left(\mathrm{~mm}^{3} / \mathrm{d}\right)$ using pooled 
animals across all treatment groups ( $n=20$ total). 9 mice ( $n=3$ per group) were randomly assigned to treatment with 1 dose of $\mathrm{DC}$ vaccines, 1 dose of $\mathrm{mDCs}$, or not reatment. 7 days after treatment, animals underwent PDAC tumor induction followed by extraction of splenocytes for LDH cytotoxicity assay. Linear regression line showed no significant correlation $\left(R^{2}=0.1259\right)$. Dashed lines show 95\% confidence around the regression line. D) Survival (d) vs. growth rate from 14-21 $\mathrm{d}\left(\mathrm{mm}^{3} / \mathrm{d}\right)$ for each treatment group ( $\mathrm{n}=5$ for each group). Linear regression was generated for each treatment or control group. Only DC-vaccines group showed a significant correlation $\left(R^{2}=0.9559, P<0.05\right)$. 

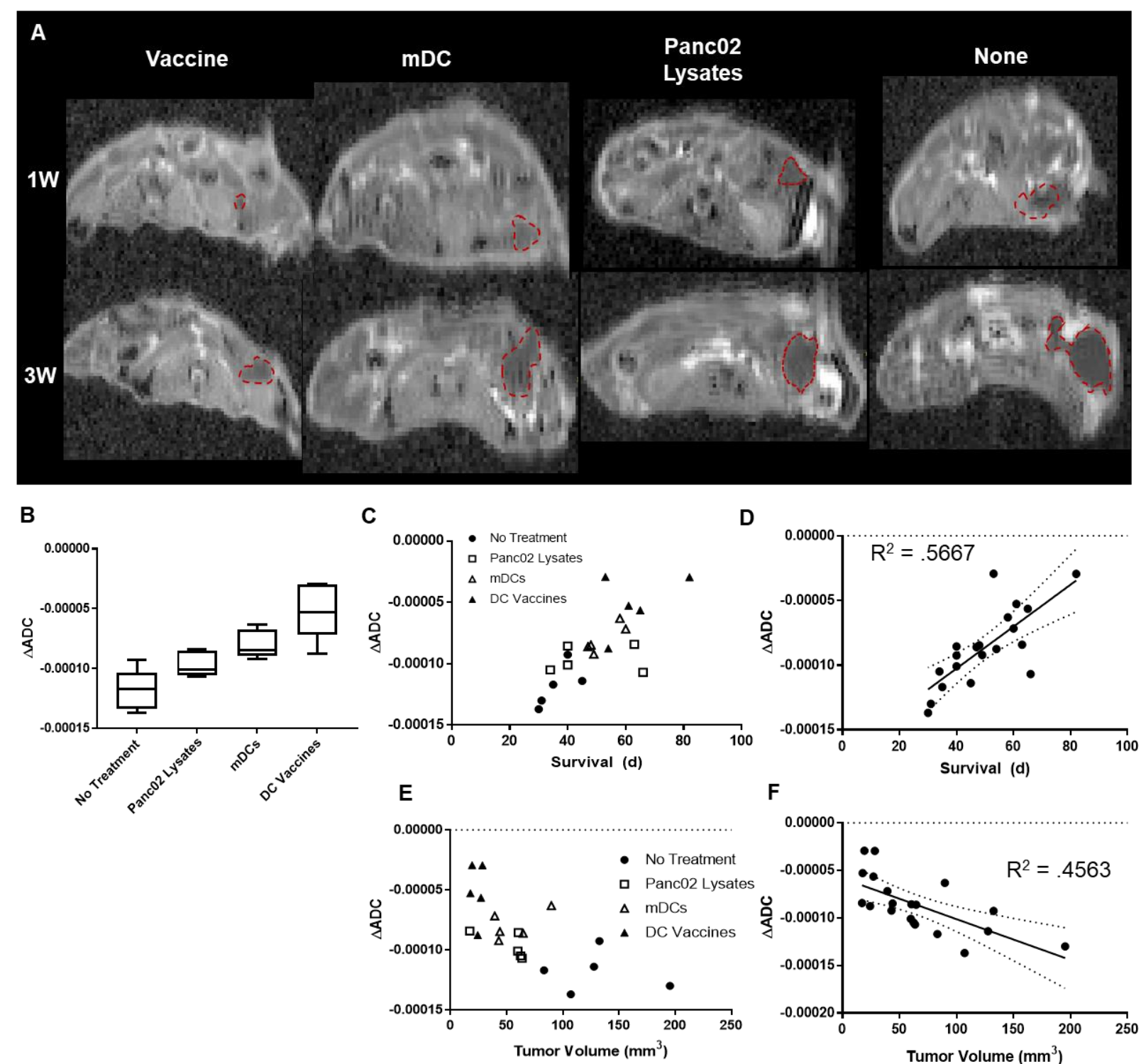

Figure 4. Representative ADC maps with additional analyses and comparisons. a) Axial ADC maps for each group ( $\mathrm{n}=5$ for each group) at 1 and 3 weeks after tumor induction. Axial images were taken at roughly the same level for each time point and experimental group. Red outline encircle the tumor on each image. b) Boxplot of tumor $\triangle A D C$ for each treatment group ( $n=5$ for each group). Entire range of $\triangle \mathrm{ADC}$ is contained between error bars. Upper, middle, and lower bounds of each box represent upper quartile, median, and lower quartile, respectively, of each group. Significant difference $(P<0.05)$ was found between sets of data from each group. $\mathbf{c}-\mathbf{d})$ $\triangle \mathrm{ADC}$ vs survival for animals across al treatment groups, separated by treatment group $(\mathbf{c}, \mathrm{n}=5$ 
for each group) or pooled for regression $(\mathbf{d}, \mathrm{n}=20)$. No distinct clustering was observed for each treatment group (c). Linear regression showed significant positive relationship between pooled survival and $\triangle A D C\left(R^{2}=0.5667, P<0.05, d\right)$. Dashed lines in $(d)$ represent $95 \%$ confidence around the regression line. e-f) $\triangle A D C$ vs tumor volume $\left(\mathrm{mm}^{3}\right)$ for each treatment group $(\mathbf{e}, \mathrm{n}=5$ for each group) or pooled across all groups (f, $n=20$ ). No distinct clustering or significant relationship was found in any treatment groups (e). Linear regression showed significant negative relationship between pooled tumor volumes $\left(\mathrm{mm}^{3}\right)$ and $\triangle A D C\left(R^{2}=0.4563, P<0.05, f\right)$. Dashed lines represent 95\% confidence around the regression line. 


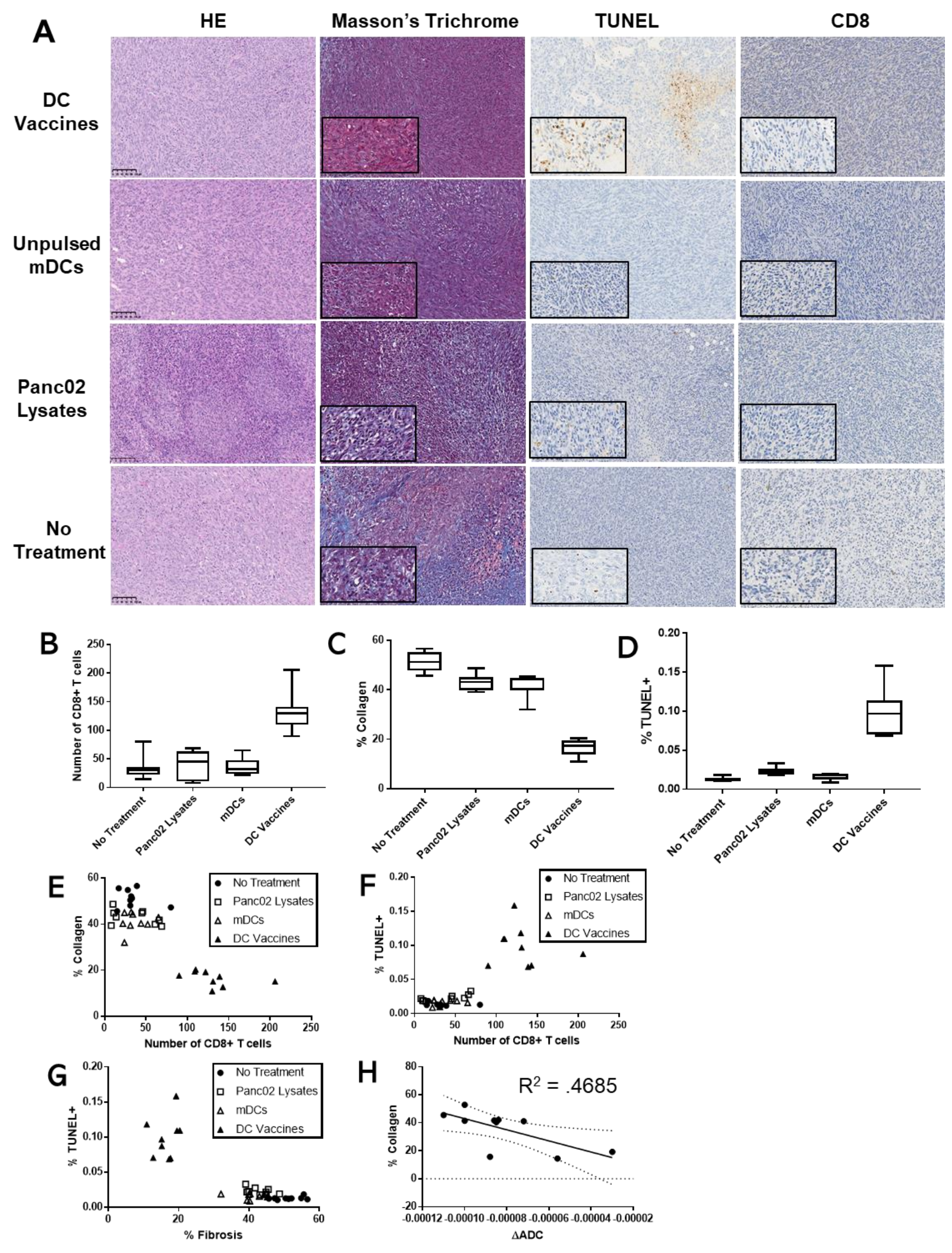


Figure 5. Histologic evaluation of tumor samples from all groups with additional analysis and comparisons to imaging measurements. a) Representative HE, Masson's Trichrome, TUNEL, and CD8 stained sections for postmortem tumor tissue samples from each treatment group. b-d) Quantitative measurements of tumor tissues from each treatment stained for $\mathrm{CD}^{+}(\mathbf{b})$, collagen (Masson's Trichrome, c), and cell apoptosis (TUNEL, d), presented as boxplots. For all boxplots, entire range of measurements for each group and stain are contained between error bars. Upper, middle, and lower bounds of the box represents upper quartile, median, and lower quartile, respectively, of each stain and treatment group. Significant differences $(P<0.05)$ were found among all treatment groups for each stain, with DC-vaccine group demonstrating the most CD8+ T cells per field, least \% collagen per field, and most \% TUNEL per field. e-g) \% collage vs number of $\mathrm{CD}^{+} \mathrm{T}$ cells $(\mathbf{e}), \%$ TUNEL vs. number of CD8 ${ }^{+} \mathrm{T}$ cells $(\mathbf{f})$, and \% TUNEL vs \% collagen $(\mathbf{g})$ for stained tumor tissue samples pooled from all treatment groups. DC vaccines clustered separately from other treatment groups in all 3 graphs. h) \% collagen vs $\triangle A D C$ for pooled tumor sample tissue across all treatment groups. Linear regression showed significant negative relationship $\left(R^{2}\right.$ $=0.04685, P<0.05)$. Dashed lines represent $95 \%$ confidence around regression line. 